\title{
Predictors of response to Gamma Knife radiosurgery for intracranial meningiomas
}

\author{
*Alireza Mansouri, MD, ${ }^{1,2}$ Soroush Larjani, BSc, ${ }^{1}$ George Klironomos, MD, PhD, 1,2 \\ Normand Laperriere, MD, FRCPC, ${ }^{3}$ Michael Cusimano MD, PhD, FRCSC, ${ }^{2,4}$ \\ Fred Gentili, MD, MSc, FRCSC,,2 Michael Schwartz, MD, MSc, FRCSC,, ${ }^{2,5}$ and \\ Gelareh Zadeh, MD, PhD, FRCSC1,2
}

\begin{abstract}
${ }^{1}$ Division of Neurosurgery, Toronto Western Hospital, University Health Network, Toronto; 'Division of Neurosurgery, University of Toronto; ${ }^{3}$ Department of Radiation Oncology, Princess Margaret Cancer Centre/University Health Network, University of Toronto; ${ }^{4}$ Keenan Research Centre, Li Ka Shing Knowledge Institute, St. Michael's Hospital, Toronto; and ${ }^{5}$ Department of Surgery (Neurosurgery), Sunnybrook Health Sciences Centre, University of Toronto, Ontario, Canada
\end{abstract}

\begin{abstract}
OBJECT In this paper, the authors' aim was to determine short-term volumetric and diametric tumor growth and identify clinical, radiological, and dosimetric predictors of adverse radiation events (AREs) following stereotactic radiosurgery (SRS) for intracranial WHO Grade I meningiomas.

METHODS This is a retrospective review of all WHO Grade I meningiomas that were treated with SRS (primary or adjuvant) between December 2005 and June 2012 at the University Health Network. Seventy-five patients had at least 24 months of both clinical and radiological follow-up and were, therefore, included in this study. Tumor growth was defined as any volumetric or diametric change greater than $10 \%$ per year. Any variation less than $+10 \%$ was considered growth stability. Volumetric measurements were made using T1-weighted gadolinium-enhanced 3-T MRI scans and ITK-SNAP software. Tumor growth rates were calculated using the specific growth rate (SGR). Univariate statistics were used to identify predictors of post-SRS AREs. All statistical analyses were performed using IBM SPSS.
\end{abstract}

RESULTS Women accounted for $69.3 \%$ of patients, and the mean treatment age was 58.6 years. Median follow-up was 36.2 months. Twenty-one (28\%) patients had undergone prior resection. Two (3\%) patients required salvage surgical intervention following SRS. The majority of the lesions (56\%) were skull base tumors. Median tumor volume and diameter were $5.2 \mathrm{~cm}^{3}$ and $27.5 \mathrm{~mm}$, respectively. The absence of tumor growth was observed in 39 cases (52\%) based on the volumetric measurements, while the absence of tumor growth was observed in 69 cases (92\%) based on the diametric measurements. Twenty-six patients (34.6\%) experienced new-onset AREs, including headache (17.3\%), cranial neuropathy $(10.6 \%)$, speech impairment $(2.7 \%)$, tremors $(2.7 \%)$, and ataxia $(1.3 \%)$. Fourteen patients $(18.7 \%)$ experienced new-onset edema, and 4 of these patients were symptomatic. A lower conformity index (1.24 vs 1.4) was significantly associated with the development of edema $(p<0.001$ power $>0.8)$. Patients with meningiomas that had growth rates of more than $10 \%$ per year were more likely to experience long-term headaches after SRS $(p=0.022)$.

CONCLUSIONS Volume-based reporting of SRS outcomes for meningiomas may be a more accurate method given the complex morphology of some lesions. The conformity index was identified as a predictor of edema following radiosurgery. http://thejns.org/doi/abs/10.3171/2014.12.JNS141687

KEY WORDS adverse event; meningioma; predictor; stereotactic radiosurgery

$\mathrm{M}$ ENINGIOMAS are the most common primary intracranial nonglial neoplasms in adults. ${ }^{34}$ When feasible, the ideal management of meningiomas is complete resection of the tumor and associated dura mater, with the goal of minimizing further neurological mor- bidity for the patient ${ }^{28}$ However, not all meningiomas are amenable to gross-total resection (e.g., extensive involvement of skull base structures or invasion into the venous sinuses or other neurovascular structures), and alternative complementary treatment strategies are necessary.,

ABBREVIATIONS ARE = adverse radiation event; $\mathrm{CI}$ RTOG = Radiation Therapy Oncology Group Conformity Index; RT = radiotherapy; SGR = specific growth rate; SRS = stereotactic radiosurgery; TVR = treatment volume ratio.

SUBMITTED July 21, 2014. ACCEPTED December 29, 2014.

INCLUDE WHEN CITING Published online July 3, 2015; DOI: 10.3171/2014.12.JNS141687.

DISCLOSURE The authors report no conflict of interest concerning the materials or methods used in this study or the findings specified in this paper.

* Dr. Mansouri and Mr. Larjani contributed equally to this work. 
While experience with conventional fractionated radiotherapy (RT) in the management of meningiomas has been greater, other modalities such as stereotactic radiosurgery (SRS) are of high utility as well. The advantage of SRS over RT is that the former allows the accurate delivery of radiation to the proposed target volume, along with a steep dosing gradient that minimizes radiation dose delivery to the surrounding structures. ${ }^{18}$ Consequently, SRS has emerged as a suitable primary/adjuvant treatment modality for the management of meningiomas, demonstrating control rates ranging from $86 \%$ to $99 \%$. $12,17,18,20,21,32,33$

While SRS minimizes many of the morbidities associated with aggressive resection, delayed adverse outcomes such as radiation necrosis, cranial nerve neuropathies, occlusion of the venous structures, hydrocephalus, and edema are possible; the occurrence of these complications is approximately $10 \%$ in the reported literature, with variability depending on the cohorts of analyzed patients. $1,31,37,43,44,46,50$ Post-SRS edema has been reported as an adverse effect/complication, which can result in neurological symptoms. Location (primarily the convexity and parasagittal regions) and larger initial tumor volume have also been associated with a higher risk of edema. . $, 17,31,50^{2}$

The variability in tumor control rates is, for the most part, attributed to the heterogeneity of the patient population, tumor histology, WHO grade, tumor size at the time of treatment, institutional variations in radiation dosing, length of follow-up, and how tumor growth and control are established. In addition, however, most methods used for reporting control rates have been restricted to $2 \mathrm{D}$ diametric measurements-where arbitrary dimensions are sometimes selected-rather than true volumetric measurements, which are potentially a more accurate reflection of the extent of tumor growth. ${ }^{3}$

In this study, we present our early institutional report of a series of 75 patients - all with solitary WHO Grade I meningiomas that were either confirmed on histopathology following resection or presumed based on the imaging appearance-who were managed with SRS between December 2005 and June 2012. Adverse radiation effects (AREs) and tumor growth rates-both diametric and volumetric-are reported. In addition, pretreatment clinical and dosimetric parameters were analyzed to identify factors predictive of the development of post-SRS AREs. Most specifically, we focused on identifying the predictors of post-SRS edema in meningiomas.

\section{Methods \\ Patient Cohort}

Between December 2005 and June 2012, 170 patients with a single intracranial WHO Grade I meningioma were treated at Universal Health Network using a model 4C Gamma Knife (Elekta Instruments). All tumors were deemed WHO Grade I meningiomas either based on histology or on the radiological appearance when resected tissue was unavailable (dura-based lesion, contrast enhancement, or the absence of signs of brain invasion and/ or edema). None of the patients had undergone prior RT. In 69 patients, the follow-up images were obtained at outside institutions and were, therefore, not amenable to analysis with the volume-contouring software used in this study; 26 patients did not have complete imaging and clinical follow-up for at least 2 years. Therefore, of the 170 treated patients, 75 were included in this study (Table 1).

This study was granted approval by the institutional research ethics board. All patients were reviewed by a multidisciplinary team of neurosurgeons and radiation oncologists prior to the initiation of treatment.

\section{Treatment Planning and Patient Follow-Up}

Treatments were planned using stereotactic CT and contrast-enhanced 1.5-mm-thick T1- and T2-weighted MRI (3-T) sequences. Our institution's practice is to include as much of the dural tail as reasonably possible within the treatment plan. Patients were clinically and radiologically followed at 3-, 6-, and 12-month intervals following the initial SRS treatment, and then yearly afterward. Imaging follow-up was obtained using T1- and T2weighted MRI sequences (1.5-T or 3-T) with a minimum slice thickness of $1.5 \mathrm{~mm}$.

\section{Analyzed Variables}

A prospectively maintained database of patients with meningiomas who were managed using SRS (primary or adjuvant) was retrospectively analyzed to obtain information regarding patient demographics (e.g., age and sex), tumor characteristics, radiation dosimetry, and new AREs attributable to SRS that arose following treatment. The frequency of steroid administration and dosing was not available for all patients and was not analyzed. The incidences of variables such as headaches and tremors were gleaned from documented subjective patient reports included with the clinical follow-up data. The studied dosimetric variables included prescription dose, prescription isodose, dose rate, treatment duration, number of isocenters, Radiation Therapy Oncology Group Conformity Index (CI RTOG) (prescription isodose volume divided by total target tumor volume), ${ }^{41}$ treatment volume ratio (TVR), homogeneity index (maximum target isodose divided by the reference isodose), maximum dose/prescribed dose within the target volume, maximum dose, minimum dose, mean dose, percentage of tumor volume receiving $100 \%$ and $95 \%$ of the prescription dose, volume receiving a $12-\mathrm{Gy}$ dose, and total target volume (Table 2). These variables were gleaned from the radiosurgery planning database.

Tumor volume was analyzed on the day of treatment and on follow-up imaging. Only MR images (pre- and posttreatment) were used for linear and volumetric measurements. Volumetric measurements were made based on the axial gadolinium-enhanced $1.5-\mathrm{mm}$ T1-weighted MRI sequences using the ITK-SNAP program (version 2.2, University of Pennsylvania). This software removes the typical assumptions used in prior volumetric measurements, where the formula approximating that of a sphere would be used (i.e., $V=4 / 3 \times \pi \times r^{3}$ ). Tumors were manually contoured by 2 authors independently and were subsequently compared for accuracy using ITK-SNAP version 2.2, which has been validated as an effective tool for measuring the volumes of anatomical structures. ${ }^{11,14,49} \mathrm{In}-$ terobserver variability was limited, demonstrating a kappa 
TABLE 1. Patient demographics and summary of the treated meningiomas

\begin{tabular}{lc}
\hline \multicolumn{1}{c}{ Parameter } & Value $^{*}$ \\
\hline Patient data & \\
\hline Mean age at treatment, yrs (range) & $58.6(24-90)$ \\
\hline Female & $52(69.3)$ \\
\hline Male & $23(30.7)$ \\
\hline Median follow-up period, mos (range) & $36.2(24.3-85.2)$ \\
\hline Prior resection & $21(28.0)$ \\
\hline Salvage resection after SRS & $2(2.7)$ \\
\hline Tumor characteristics & $5.2(0.7-25.8)$ \\
\hline Median tumor vol, cm ${ }^{3}$ (range) & $27.5(13.0-56.5)$ \\
\hline Median tumor diameter, mm (range) & $14(19 \%)$ \\
\hline Convexity & $8(11 \%)$ \\
\hline Falcotentorial & $12(16 \%)$ \\
\hline Parasagittal & $9(12 \%)$ \\
\hline Anterior/middle fossa base & $9(12 \%)$ \\
\hline Posterior fossa & $4(5 \%)$ \\
\hline Cavernous sinus & $18(24 \%)$ \\
\hline Cerebellopontine angle/petroclival & $1(1 \%)$ \\
\hline Intraventricular & \\
\hline
\end{tabular}

* Values are number of patients (\%) unless otherwise specified.

value of 0.94 . Similar to most other studies, the value used to calculate the "diameter" of the tumor for comparisons with the linear measurements was the maximal diameter in any given cardinal plane.

Tumor growth rates were calculated using the specific growth rate $(\mathrm{SGR})$ equation: ${ }^{25} \mathrm{SGR}=\ln (V 2 / V 1) /(t 2-t 1) \times$ $100 \%$, where $V$ represents the tumor volume in cubic centimeters at a given time point, and $t$ represents the date MRI was performed. The growth rate was calculated between the MR images obtained on the treatment date and 12 months post-SRS in order to calculate the tumor growth rate per year following treatment. In addition, to ensure that the growth rate in the 1st year following treatment was not due to pseudoprogression, growth rates were also calculated between the treatment date and 24 months post-SRS. The average of the 2 time points is presented as the annual growth rate for each case. Tumor growth was defined as any volumetric or diametric change greater than $10 \%$ per year in order to account for possible pseudoprogression and errors in measurement. ${ }^{11,39}$ Any variation less than $+10 \%$ was considered growth stability. Traditional methods for measuring tumor growth are either diametric or volumetric, whereby the tumor's greatest diameter in any given cardinal plane is assessed over time. ITK-SNAP software enables the accurate calculation of the tumor volume by addressing 2 key flaws in the aforementioned approaches. The first flaw of the traditional methods is the false assumption that the morphology of a tumor is approximated by a sphere, which is incorrect. The second flaw is that using the measurements of a single diametric plane assumes that the tumor only grows in 1 dimension and 1 plane. Based on this latter assumption, a tumor that has grown by only $+10 \%$ in diameter but in every dimension-which cannot be ascertained as true or false using
TABLE 2. Dosimetric parameters evaluated as potential predictors of adverse events

\begin{tabular}{lc}
\hline Dosimetric Characteristics & Mean (range) \\
\hline No. of isocenters & $14.9(1.0-42.0)$ \\
\hline Prescription isodose, Gy & $48.0(20.0-80.0)$ \\
\hline Total treatment time, mins & $53.5(2.7-183.2)$ \\
\hline Dose rate, Gy/min & $3.0(1.6-3.5)$ \\
\hline Prescription dose, mean Gy & $13.0(12.0-16.0)$ \\
\hline CI RTOG & $1.4(1.1-2.1)$ \\
\hline MDPD & $2.1(1.3-5.0)$ \\
\hline TVR & $0.7(0.5-0.9)$ \\
\hline Target min dose, Gy & $10.2(5.2-16.9)$ \\
\hline Target max dose, Gy & $28.0(15.0-80.0)$ \\
\hline Target mean dose, Gy & $18.8(13.6-27.1)$ \\
\hline
\end{tabular}

MDPD = maximum dose/prescribed dose within the target volume

single diametric measurements-would correspondingly have a volumetric change of approximately $+33 \%$. By providing a sum of the surface area values of the manually contoured outlines of the lesion in each plane, ITK-SNAP software calculates an accurate overall volumetric value that corresponds to the true morphology of the tumor without any assumptions.

The AREs noted in the literature that are attributable to SRS include headaches, cranial neuropathy, seizure, speech

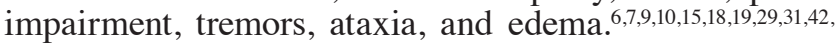
44,50 Therefore, the new onset of any of the aforementioned complications in each patient following SRS was documented and analyzed. The development of any new T2 or FLAIR signal changes surrounding the lesion following SRS were interpreted as edema.

\section{Statistical Analysis}

Logistic regression analysis was used to test for any significant differences in radiation dosimetry between patients who did and did not experience post-SRS AREs. Linear regression was used to investigate correlations between radiation dosimetry and tumor growth rates after SRS; $p$ values $<0.05$ were considered significant. The power was also determined for statistical tests with significant $\mathrm{p}$ values, and power $>0.8$ was considered significant. All analyses were performed using IBM SPSS (version 20.0, IBM SPSS).

\section{Results}

\section{Patient and Tumor Characteristics}

Clinical and radiological data were collected and evaluated for the 75 patients with WHO Grade I intracranial meningiomas (Table 1). Our cohort consisted of 52 female $(69.3 \%)$ and 23 male $(30.7 \%)$ patients. The median followup period after SRS was 36.2 months (range 24.3-85.2 months). The mean age at treatment was 58.6 years (range 24-90 years). Twenty-one patients (28\%) had undergone prior resection and were treated with SRS for tumor recurrence. The remaining 54 patients (72\%) received SRS as the primary treatment. Only 2 patients (3\%) experienced significant symptomatic tumor growth after SRS that re- 
quired salvage microsurgical resection; none were secondary to symptomatic edema. The median treated tumor volume was $5.2 \mathrm{~cm}^{3}$ (range $0.7-25.8 \mathrm{~cm}^{3}$ ), and the median largest tumor diameter was $27.5 \mathrm{~mm}$ (range 13.0-56.4 $\mathrm{mm})$. Forty-two (56\%) tumors were within the skull base, while the remaining tumors were nonbasal. Among the 35 nonbasal tumors, 20 (57\%) were falcotentorial/parasagittal, $14(40 \%)$ were in the convexity, and $1(3 \%)$ was intraventricular.

\section{Tumor Growth Rates}

Thirty-nine $(52.0 \%)$ patients demonstrated stable tumor size, based on the volumetric measurements, and an average growth rate of $-10.7 \%$ per year. The remaining $36(48.0 \%)$ meningiomas demonstrated an average growth rate of $+42.7 \%$ per year following SRS. Using 2D measurements, on the other hand, 69 patients (92\%) demonstrated tumor growth stability with an average growth rate of $-5.8 \%$ per year. The remaining 6 patients $(8 \%)$ were found to have an average growth rate of $+20 \%$ per year.

\section{Adverse Radiation Effects}

Twenty-six patients $(34.6 \%)$ experienced some form of new-onset AREs following SRS (Fig. 1). AREs such as stroke, seizure, or hydrocephalus were not observed. Eight patients $(10.6 \%)$ developed some form of cranial neuropathy, with the most common form being trigeminal dysfunction in 5 of 8 patients. In all 5 patients with trigeminal neuropathy, the meningiomas were basal. While variable depending on tumor location, the mean dose delivered to the brainstem, optic apparatus, trigeminal nerve, and cranial nerves VII and VIII were $11.8 \mathrm{~Gy}, 3.8 \mathrm{~Gy}, 11.2 \mathrm{~Gy}$, and $8.1 \mathrm{~Gy}$, respectively.

Fourteen patients (18.7\%) were found to have new T2/ FLAIR signal changes following SRS that were suggestive of edema. Four of these patients $(2.9 \%)$ were symptomatic, and all presented with headaches. The volumes of the meningiomas in the patients with or without evidence of edema were $6.7 \mathrm{~cm}^{3}$ and $4.9 \mathrm{~cm}^{3}$, respectively. With regard to the correlation between tumor location and edema, 2 tumors were parasagittal, 3 were falcotentorial, 3 were in the frontal convexity, and 6 were basal (of which 1 was in the cavernous sinus).

Patients with meningiomas that showed significant tumor growth following SRS (i.e., greater than $10 \%$ per year) were more likely to experience long-term headaches after SRS $(p=0.022)$ The post-SRS tumor growth rate, however, was not a predictor of other AREs. Based on univariate analysis, the development of radiological T2/ FLAIR changes that were suggestive of edema was found to be significantly associated with lower CI RTOG (1.24 vs 1.4 ) following SRS ( $p<0.001$, power $>0.8$ ) (Table 3$)$. Lesion location and volume were not found to be significant predictors of edema. Given the insufficient power of this study, a multivariate analysis was not performed.

\section{Discussion}

SRS is a noninvasive and effective therapeutic modality for intracranial meningiomas that has been used as a primary and adjuvant therapy for lesions in the central nervous system for the last 30 years. Many studies have evalu-

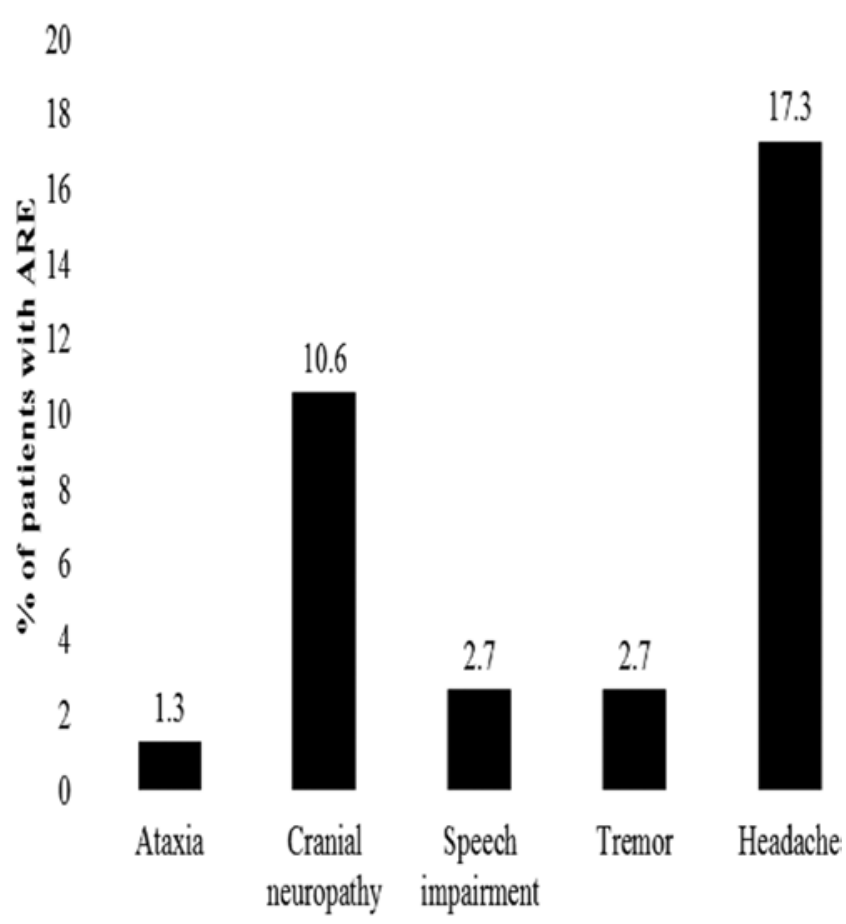

FIG. 1. Breakdown of post-SRS AREs. Clinically determined adverse events that were documented in our patients over a minimum follow-up of 2 years.

ated the efficacy of SRS for treating meningiomas and reported outcomes and common adverse effects., $, 9,16,22,24,31,44$ These retrospective studies suggest local control rates between $86 \%$ and $99 \%$, tumor regression rates up to $70 \%$, symptom improvement in $8 \%$ to $65 \%$ of cases, and ARE rates of $2.5 \%$ to $43 \%$. In the current early follow-up study of patients with WHO Grade I meningiomas who underwent SRS, we demonstrate the disparity between volumetric and linear measurements with regard to tumor growth. In addition, we report an inclusive list of AREs and identified dosimetric parameters that are potentially predictive of these AREs.

\section{Tumor Growth Rate}

Using diametric measurements, $92 \%$ of the tumors in our study remained stable in size-based on our definition of growth $<+10 \%$ - which is within the range reported by various groups in the literature that used various definitions of growth stability. ${ }^{12,17,18,20,32}$ However, using volumetric or 3D measurements, only $52 \%$ of tumors demonstrated growth stability. This great disparity highlights the flawed conclusions that could possibly be derived from simply assessing diametric growth. In this early follow-up study, we have demonstrated that there is a large disparity between our 2-year volumetric and diametric measurements of tumor growth. Given the 2-year time frame, this cannot be considered a report on the long-term tumor control rates. Nonetheless, this difference supports the limitations of using linear measurements for accurately tracking tumor response and, thus, we believe future studies on treatment outcomes would benefit from supplementing their data with volumetric measurements as well. Volumetric measurements were used to quantify tumor control in a recent 


\begin{tabular}{|c|c|c|c|}
\hline \multicolumn{2}{|c|}{$\begin{array}{l}\text { Univariate Analysis Between Post-SRS } \\
\text { Complications and Dosimetric Variables }\end{array}$} & \multicolumn{2}{|c|}{ Statistical Significance } \\
\hline $\begin{array}{l}\text { Post-SRS } \\
\text { Complication }\end{array}$ & Dosimetric Variable & $p$ Value & Power (\%) \\
\hline Ataxia & Prescription dose (Gy) & $<0.001$ & 19.2 \\
\hline Tremors & Prescription dose (Gy) & $<0.001$ & 19.2 \\
\hline Edema & CIRTOG & $<0.001$ & 85.7 \\
\hline Edema & TVR & $<0.001$ & 92.7 \\
\hline
\end{tabular}

large, multicenter, European study on benign meningiomas that were managed using GKRS..$^{39}$ The control rate in that study was defined in the same way as our study, and it was reported to be $92.5 \%$. A comparison with control rates based on 2D measurements in the same cohort was not made. While that study contained a large number of patients who were followed over an extended period of time, the volumetric measurement methods were heterogeneous: both CT and MRI measurements were considered, and in cases in which digital images were not available, calculations were made using measurements on celluloid films. In our study, all measurements were made using digital T1-weighted gadolinium-enhanced MRI sequences and the same software (ITK SNAP version 2.2); furthermore, these measurements were confirmed in a blinded manner in duplicate with good correlation between $2 \mathrm{ob}$ servers. Regardless of these technical differences, the work of Santacroce et al. ${ }^{39}$ along with our current study, highlights the fact that 3D volume measurements, while technically more demanding, are a more accurate reflection of the morphological identity of the tumor and, hence, a potentially better method for reporting clinical response. Therefore, we advocate that future studies assess tumor control rates in any neoplastic lesion and tumor volume rather than the greatest $2 \mathrm{D}$ measurements. Furthermore, increased accuracy can be achieved with the aid of volume-measuring software over prior methods which have deduced the volume based on the largest dimensions in 3 planes.

\section{Adverse Radiation Effects}

Overall, $34.6 \%$ of patients treated with SRS in our study developed some form of posttreatment ARE such as headaches, cranial neuropathies, or ataxia.

The development of new imaging-based post-SRS edema was observed in $18.7 \%$ of treated patients in our study; there was no statistically significant correlation between tumor location or volume and the incidence of edema. Only 4 of 14 patients were symptomatic, all of whom experienced headaches. Patients with a lower CI RTOG (1.24 vs 1.4$)$ were found to be at a significantly higher risk of developing imaging-based edema after SRS. The development of edema is a well-known ARE following SRS. ${ }^{2,20,23,31,47,50}$ While meningioma location within the convexity or parasagittal regions, larger tumor volume, and greater radiation dosing appear to be factors that are often predictive of an increased risk of edema, none of these variables were found to be significant in our study. ${ }^{2,31,50}$ In the study by Patil et al., where edema was similarly defined based on new T2 signal changes on MRI, close to one-third of patients harbored meningiomas that were within the parasagittal region. Similarly, Chang et al. $^{2}$ demonstrated that tumor location within the convexity region was significantly predictive of post-SRS T2 signal changes. Zada et al., ${ }^{50}$ using a similar definition of edema, showed that lesion volume was almost double in patients who developed post-SRS edema in comparison with patients without SRS-edema (11 vs $\left.5.7 \mathrm{~cm}^{3}\right)$. The incidence of edema in our study, however, was similar regardless of tumor location (basal or nonbasal). While the meningiomas in the patients with evidence of edema were larger, the average size of these lesions was smaller than those reported in previous studies that examined the correlation between size and adverse outcomes, which could have contributed to the absence of statistical significance in our study. ${ }^{18,30}$ As a result, CI RTOG emerged as a potentially significant predictor of edema in our study, which warrants further analysis and verification.

The correlation between a lower CI value and an increased risk of edema is rather peculiar and worthy of further discussion. CI RTOG is defined as the volume covered by the prescription isodose divided by the total target tumor volume. ${ }^{45}$ Mathematically, perfect overlap between the targeted volume and total tumor volume would correspond to a CI value of 1.0. On the other hand, decreasing the volume of coverage or increasing the lesion volume would result in a lower CI value. In a study by Wu et al., larger lesions were calculated to have a smaller conformity value regardless of the type of treatment plan. Among large-volume lesions, plans with mixed dose coverage (i.e., overcoverage in some segments and undercoverage in others) also had lower conformity values. ${ }^{48}$ In addition, CI RTOG does not take into consideration the quality of coverage: ${ }^{8}$ in theory, the target volume may be the same as the lesion volume without any overlap of the 2 volumes. While this is an extreme example, the issue is indeed particularly relevant to areas of debate such as coverage of the dural tail in exchange for lower conformity (i.e., higher CI value). ${ }^{5,38}$ Therefore, within the spectrum of RTOG-acceptable treatment plans, it is nonetheless possible to observe adverse events secondary to the unique characteristics of the lesion(s) or other aspects of the radiosurgical plan. On the other hand, it is very likely that the development of edema is linked to the biological components of the tumor rather than solely radiation parameters. The expression of molecules such as CEA (carcinoembryonic antigen), CK (creatine kinase), VEGF (vascular endothelial growth factor), and HIF-1 (hypoxia-inducible factor) has been shown to be predictive of the development of edema in meningiomas following radiosurgery. ${ }^{13,36}$ In the current study, the correlation between the CI RTOG value and the presence of edema is likely attributable to the combination of lesion shape irregularities, our attempt to provide dose coverage to the dural tail while minimizing irradiation to healthy brain tissue (thereby resulting in an absence of coverage in certain areas of the dural tail), and the underlying biology of the lesions.

Headaches accounted for the highest proportion of adverse events noted in our study population. However, giv- 
en the subjective nature of headaches, it was not possible to determine if these AREs were purely associated with SRS or if the presence and/or growth of the tumor was a contributory factor as well. Patients demonstrating growth rates greater than $10 \%$ per year were significantly more likely to develop nonspecific headaches. While this can be attributed to increased intracranial pressure, the aggressive nature of these lesions-based on their rapid growth-resulting in irritation of the dural lining is also a possibility.

The majority of the cranial neuropathies were attributable to worsening or new-onset trigeminal neuralgia; the trigeminal nerve is one of the nerves that is most commonly affected when SRS is used to manage intracranial meningiomas. ${ }^{26,44}$ In all 5 cases of trigeminal neuropathy included in this study, the treated meningioma was located in the cerebellopontine angle. At an average dose of 11.2 $\mathrm{Gy}$, the extent of radiation administered to the trigeminal nerve in our series was well below the previously suggested limit of $19 \mathrm{~Gy}^{26}$ The incidence of cranial neuropathies was too low to identify the dosimetric parameters that would be significant predictors of this event. Other disabling AREs identified in previous studies, such as seizure and stroke, were not observed in our study. 35

Our study is limited by the relatively short follow-up period, and, as such, conclusions are relevant to the shortterm period following SRS in meningiomas. Furthermore, given the tertiary referral nature of our center, many patients underwent follow-up at their home institution; therefore, although we treated 170 patients, only 75 patients had complete clinical and radiological follow-up at 2 years and imaging performed at local institutions was not available for review. In addition, the retrospective nature of this study implies that potentially important dosimetric and clinical variables may have been omitted or incompletely collected, thus limiting our analysis. Given the short time frame, the goal of the current study is not to report our institutional tumor control rate, but rather to present concerns regarding the disparity between traditional methods for reporting tumor growth in comparison with more tedious but accurate volumetric measurement method. More durable conclusions regarding the tumor control rate and adverse outcomes could be attained if patients were followed for a longer period of time. Nonetheless, our study is the first to assess the volumetric growth of meningiomas following SRS using a uniform and replicable technique, thereby demonstrating this large disparity between volume- and diameter-based growth measurements. In addition, we show an association between post-SRS edema and the lower CI RTOG range that is within guidelines. While this may be a function of the quality of SRS planning, the biology of the individual lesions cannot be ignored. Future studies aimed at assessing the validity of this correlation and potential explanations for this trend would be of high value.

\section{Conclusions}

We demonstrate that CI ROTG can be a predictor of edema following SRS. We also demonstrate that while the short-term trends for tumor growth based on linear measurements in this study are similar to the long-term control rates reported by prior studies, volume-based measure- ments do not demonstrate as favorable a tumor control rate. Thus, we recommend that future studies report tumor control rates based on volumetric measurements.

\section{References}

1. Bloch O, Kaur G, Jian BJ, Parsa AT, Barani IJ: Stereotactic radiosurgery for benign meningiomas. J Neurooncol 107:13-20, 2012

2. Chang JH, Chang JW, Choi JY, Park YG, Chung SS: Complications after gamma knife radiosurgery for benign meningiomas. J Neurol Neurosurg Psychiatry 74:226-230, 2003

3. Cusimano MD, Kan P, Nassiri F, Anderson J, Goguen J, Vanek I, et al: Outcomes of surgically treated giant pituitary tumours. Can J Neurol Sci 39:446-457, 2012

4. Cusimano MD, Sekhar LN, Sen CN, Pomonis S, Wright DC, Biglan AW, et al: The results of surgery for benign tumors of the cavernous sinus. Neurosurgery 37:1-10, 1995

5. DiBiase SJ, Kwok Y, Yovino S, Arena C, Naqvi S, Temple $\mathrm{R}$, et al: Factors predicting local tumor control after gamma knife stereotactic radiosurgery for benign intracranial meningiomas. Int J Radiat Oncol Biol Phys 60:1515-1519, 2004

6. Duma CM, Lunsford LD, Kondziolka D, Bissonette DJ, Somaza S, Flickinger JC: Radiosurgery for vascular malformations of the brain stem. Acta Neurochir Suppl (Wien) 58:92-97, 1993

7. El-Khatib M, El Majdoub F, Hoevels M, Kocher M, Müller RP, Steiger HJ, et al: Stereotactic LINAC radiosurgery for incompletely resected or recurrent atypical and anaplastic meningiomas. Acta Neurochir (Wien) 153:1761-1767, 2011

8. Feuvret L, Noël G, Mazeron JJ, Bey P: Conformity index: a review. Int J Radiat Oncol Biol Phys 64:333-342, 2006

9. Girvigian MR, Chen JC, Rahimian J, Miller MJ, Tome M: Comparison of early complications for patients with convexity and parasagittal meningiomas treated with either stereotactic radiosurgery or fractionated stereotactic radiotherapy. Neurosurgery 62 (5 Suppl):A19-A28, 2008

10. Hayhurst C, Monsalves E, van Prooijen M, Cusimano M, Tsao M, Menard C, et al: Pretreatment predictors of adverse radiation effects after radiosurgery for arteriovenous malformation. Int J Radiat Oncol Biol Phys 82:803-808, 2012

11. Hayhurst C, Zadeh G: Tumor pseudoprogression following radiosurgery for vestibular schwannoma. Neuro Oncol 14:87-92, 2012

12. Igaki H, Maruyama K, Koga T, Murakami N, Tago M, Terahara A, et al: Stereotactic radiosurgery for skull base meningioma. Neurol Med Chir (Tokyo) 49:456-461, 2009

13. Kan P, Liu JK, Wendland MM, Shrieve D, Jensen RL: Peritumoral edema after stereotactic radiosurgery for intracranial meningiomas and molecular factors that predict its development. J Neurooncol 83:33-38, 2007

14. Kelly JP, Weiss AH: Detection of tumor progression in optic pathway glioma with and without neurofibromatosis type 1 . Neuro Oncol 15:1560-1567, 2013

15. Kim DG, Kim ChH, Chung HT, Paek SH, Jeong SS, Han DH, et al: Gamma knife surgery of superficially located meningioma. J Neurosurg 102 Suppl:255-258, 2005

16. Kollová A, Liscák R, Novotný J Jr, Vladyka V, Simonová G, Janousková L: Gamma Knife surgery for benign meningioma. J Neurosurg 107:325-336, 2007

17. Kondziolka D, Flickinger JC, Perez B: Judicious resection and/or radiosurgery for parasagittal meningiomas: outcomes from a multicenter review. Neurosurgery 43:405-414, 1998

18. Kondziolka D, Levy EI, Niranjan A, Flickinger JC, Lunsford LD: Long-term outcomes after meningioma radiosurgery: physician and patient perspectives. J Neurosurg 91:44-50, 1999

19. Kondziolka D, Madhok R, Lunsford LD, Mathieu D, Martin JJ, Niranjan A, et al: Stereotactic radiosurgery for convexity meningiomas. J Neurosurg 111:458-463, 2009 
20. Kondziolka D, Mathieu D, Lunsford LD, Martin JJ, Madhok R, Niranjan A, et al: Radiosurgery as definitive management of intracranial meningiomas. Neurosurgery 62:53-60, 2008

21. Korah MP, Nowlan AW, Johnstone PA, Crocker IR: Radiation therapy alone for imaging-defined meningiomas. Int J Radiat Oncol Biol Phys 76:181-186, 2010

22. Kreil W, Luggin J, Fuchs I, Weigl V, Eustacchio S, Papaefthymiou G: Long term experience of gamma knife radiosurgery for benign skull base meningiomas. J Neurol Neurosurg Psychiatry 76:1425-1430, 2005

23. Lee SR, Yang KA, Kim SK, Kim SH: Radiation-induced intratumoral necrosis and peritumoral edema after gamma knife radiosurgery for intracranial meningiomas. J Korean Neurosurg Soc 52:98-102, 2012

24. Malik I, Rowe JG, Walton L, Radatz MW, Kemeny AA: The use of stereotactic radiosurgery in the management of meningiomas. Br J Neurosurg 19:13-20, 2005

25. Mehrara E, Forssell-Aronsson E, Ahlman H, Bernhardt P: Specific growth rate versus doubling time for quantitative characterization of tumor growth rate. Cancer Res 67:39703975, 2007

26. Morita A, Coffey RJ, Foote RL, Schiff D, Gorman D: Risk of injury to cranial nerves after gamma knife radiosurgery for skull base meningiomas: experience in 88 patients. J Neurosurg 90:42-49, 1999

27. Naumann M, Meixensberger J: Factors influencing meningioma recurrence rate. Acta Neurochir (Wien) 107:108-111, 1990

28. Newman SA: Meningiomas: a quest for the optimum therapy. J Neurosurg 80:191-194, 1994

29. Nicolato A, Foroni R, Alessandrini F, Bricolo A, Gerosa M: Radiosurgical treatment of cavernous sinus meningiomas: experience with 122 treated patients. Neurosurgery 51:11531161,2002

30. Ojemann SG, Sneed PK, Larson DA, Gutin PH, Berger MS, Verhey L, et al: Radiosurgery for malignant meningioma: results in 22 patients. J Neurosurg 93 (Suppl 3):62-67, 2000

31. Patil CG, Hoang S, Borchers DJ III, Sakamoto G, Soltys SG, Gibbs IC, et al: Predictors of peritumoral edema after stereotactic radiosurgery of supratentorial meningiomas. Neurosurgery 63:435-442, 2008

32. Pollock BE: Stereotactic radiosurgery for intracranial meningiomas: indications and results. Neurosurg Focus 14(5):e4, 2003

33. Pollock BE, Stafford SL: Results of stereotactic radiosurgery for patients with imaging defined cavernous sinus meningiomas. Int J Radiat Oncol Biol Phys 62:1427-1431, 2005

34. Pollock BE, Stafford SL, Link MJ, Brown PD, Garces YI, Foote RL: Single-fraction radiosurgery of benign intracranial meningiomas. Neurosurgery 71:604-613, 2012

35. Pollock BE, Stafford SL, Link MJ, Garces YI, Foote RL: Stereotactic radiosurgery of World Health Organization grade II and III intracranial meningiomas: treatment results on the basis of a 22-year experience. Cancer 118:1048-1054, 2012

36. Regelsberger J, Hagel C, Emami P, Ries T, Heese O, Westphal M: Secretory meningiomas: a benign subgroup causing life-threatening complications. Neuro Oncol 11:819-824, 2009

37. Roche PH, Régis J, Dufour H, Fournier HD, Delsanti C, Pellet W, et al: Gamma knife radiosurgery in the management of cavernous sinus meningiomas. J Neurosurg 93 (Suppl 3):68-73, 2000

38. Rogers L, Jensen R, Perry A: Chasing your dural tail: Factors predicting local tumor control after gamma knife stereotactic radiosurgery for benign intracranial meningiomas. Int J Radiat Oncol Biol Phys 62:616-619, 2005 (Letter)

39. Santacroce A, Walier M, Régis J, Liščák R, Motti E,
Lindquist C, et al: Long-term tumor control of benign intracranial meningiomas after radiosurgery in a series of 4565 patients. Neurosurgery 70:32-39, 2012

40. Sekhar LN, Patel S, Cusimano M, Wright DC, Sen CN, Bank WO: Surgical treatment of meningiomas involving the cavernous sinus: evolving ideas based on a ten year experience. Acta Neurochir Suppl 65:58-62, 1996

41. Shaw E, Kline R, Gillin M, Souhami L, Hirschfeld A, Dinapoli R, et al: Radiation Therapy Oncology Group: radiosurgery quality assurance guidelines. Int J Radiat Oncol Biol Phys 27:1231-1239, 1993

42. Shin M, Kurita H, Sasaki T, Kawamoto S, Tago M, Kawahara $\mathrm{N}$, et al: Analysis of treatment outcome after stereotactic radiosurgery for cavernous sinus meningiomas. J Neurosurg 95:435-439, 2001

43. Skeie BS, Enger PO, Skeie GO, Thorsen F, Pedersen PH: Gamma knife surgery of meningiomas involving the cavernous sinus: long-term follow-up of 100 patients. Neurosurgery 66:661-669, 2010

44. Stafford SL, Pollock BE, Foote RL, Link MJ, Gorman DA, Schomberg PJ, et al: Meningioma radiosurgery: tumor control, outcomes, and complications among 190 consecutive patients. Neurosurgery 49:1029-1038, 2001

45. Stanley J, Breitman K, Dunscombe P, Spencer DP, Lau H: Evaluation of stereotactic radiosurgery conformity indices for 170 target volumes in patients with brain metastases. J Appl Clin Med Phys 12:3449, 2011

46. Tishler RB, Loeffler JS, Lunsford LD, Duma C, Alexander E III, Kooy HM, et al: Tolerance of cranial nerves of the cavernous sinus to radiosurgery. Int $\mathbf{J}$ Radiat Oncol Biol Phys 27:215-221, 1993

47. Unger KR, Lominska CE, Chanyasulkit J, Randolph-Jackson P, White RL, Aulisi E, et al: Risk factors for posttreatment edema in patients treated with stereotactic radiosurgery for meningiomas. Neurosurgery 70:639-645, 2012

48. Wu QR, Wessels BW, Einstein DB, Maciunas RJ, Kim EY, Kinsella TJ: Quality of coverage: conformity measures for stereotactic radiosurgery. J Appl Clin Med Phys 4:374-381, 2003

49. Yushkevich PA, Detre JA, Tang KZ, Hoang A, MechanicHamilton D, Fernandez-Seara MA, et al: Hippocampusspecific fMRI group activation analysis with continuous M-Reps. Med Image Comput Comput Assist Interv 9:284-291, 2006

50. Zada G, Pagnini PG, Yu C, Erickson KT, Hirschbein J, Zelman V, et al: Long-term outcomes and patterns of tumor progression after gamma knife radiosurgery for benign meningiomas. Neurosurgery 67:322-329, 2010

\section{Author Contributions}

Conception and design: Mansouri, Zadeh. Acquisition of data: Larjani, Klironomos. Analysis and interpretation of data: Mansouri, Larjani. Drafting the article: Mansouri. Critically revising the article: Mansouri. Reviewed submitted version of manuscript: all authors. Statistical analysis: Mansouri, Larjani, Klironomos. Administrative/technical/material support: Mansouri, Study supervision: Laperriere, Cusimano, Gentili, Schwartz, Zadeh.

\section{Correspondence}

Alireza Mansouri, Division of Neurosurgery, Toronto Western Hospital, 4W-436, 399 Bathurst St., Toronto, ON M5T 2S8, Canada. email: alireza.mansouri@utoronto.ca. 